\title{
Ignition conditions for inertial confinement fusion targets with a nuclear spin-polarized DT fuel
}

\author{
M. Temporal ${ }^{1}$, V. Brandon ${ }^{2}$, B. Canaud ${ }^{2}$, J.P. Didelez ${ }^{3}$, \\ R. Fedosejevs ${ }^{4}$ and R. Ramis ${ }^{1}$ \\ ${ }^{1}$ ETSIA, Universidad Politécnica de Madrid, 28040 Madrid, Spain \\ ${ }^{2}$ CEA, DAM, DIF, F-91297 Arpajon, France \\ ${ }^{3}$ Institut de Physique Nucléaire, CNRS/IN2P3 et Université Paris XI, 91406 Orsay, France \\ ${ }^{4}$ Department of Electrical and Computer Engineering, University of Alberta, Edmonton, \\ AB T6G 2V4, Canada \\ E-mail: mauro.temporal@hotmail.com
}

\begin{abstract}
The nuclear fusion cross-section is modified when the spins of the interacting nuclei are polarized. In the case of deuterium-tritium it has been theoretically predicted that the nuclear fusion cross-section could be increased by a factor $\delta=1.5$ if all the nuclei were polarized. In inertial confinement fusion this would result in a modification of the required ignition conditions. Using numerical simulations it is found that the required hot-spot temperature and areal density can both be reduced by about $15 \%$ for a fully polarized nuclear fuel. Moreover, numerical simulations of a directly driven capsule show that the required laser power and energy to achieve a high gain scale as $\delta^{-0.6}$ and $\delta^{-0.4}$ respectively, while the maximum achievable energy gain scales as $\delta^{0.9}$.
\end{abstract}

(Some figures may appear in colour only in the online journal)

\section{Introduction}

For many years it has been known that the deuteriumtritium (DT) nuclear fusion cross-section [1] depends on the spin polarization of the interacting nuclei [2]. The case of DT thermonuclear fusion fuel is of special interest in both approaches to controlled fusion energy: magnetic confinement fusion (MCF) [3] and inertial confinement fusion (ICF) [4-8]. For the $\mathrm{D}(\mathrm{T}, \mathrm{n})^{4} \mathrm{He}$ nuclear fusion reaction the theory predicts a maximum increase in the nuclear fusion cross-section of a factor $3 / 2$. In fact, by assuming that the fractions of the deuterium ( $s=1)$ and tritium $(s=1 / 2)$ spins with respect to the $z$-direction are $\mathrm{d}_{+}, \mathrm{d}_{0}, \mathrm{~d}_{-}$and $\mathrm{t}_{+}, \mathrm{t}_{-}$, it follows [2] that the total fusion cross-section is given by $\sigma=(3 / 2 a+b+c / 2) \sigma_{0}=$ $\delta \sigma_{0}$, where $a=\mathrm{d}_{+} \mathrm{t}_{+}+\mathrm{d}_{-} \mathrm{t}_{-}, b=d_{0}, c=\mathrm{d}_{+} \mathrm{t}_{-}+\mathrm{d}_{-} \mathrm{t}_{+}$ and $\sigma_{0}$ is the cross-section for unpolarized nuclei. Thus, for a fully polarized DT fuel ( $a=1, b=c=0$ ) the fusion cross-section increases by a factor $\delta=1.5$ with respect to the unpolarized fuel. At the same time, the particles produced by the fusion reactions are characterized by anisotropy in angular distribution [2], which could be advantageous in controlling the effect of neutrons on the reactor components.

The effect of the spin polarization of the nuclei of interest for fusion reactions has been investigated theoretically
[2,9-11], and a recent summary of the status of polarized fusion has been given by Paetz gen. Schieck [12]. The polarization of DT fuel is technologically challenging. For MCF, polarized gas can be used and atomic beams of $\mathrm{H}$ and $\mathrm{D}$ are currently produced with high polarization. There has been significant progress in the development of atomic beam sources (ABSs) over the last few decades. The most intense ABS until now is used at the Brookhaven's Relativistic Heavy Ion Collider which is able to deliver a polarized beam of up to $8 \times 10^{16}$ atoms s$^{-1}$ [13]. Moreover, the ABS of the Hermes experiment at DESY (Hamburg) and the ABS for the magnetic spectrometer at the Cooler Synchrotron (COSY, Jülich) can produce deuterium beams with intensities of up to $6 \times 10^{16}$ atoms s $^{-1}$ [14]. It has also been proposed to polarize hydrogen molecules, which could produce molecular beams with a much higher intensity of $10^{18}$ molecules $s^{-1}$ [15]. In the case of ICF, cryogenic targets are required. The symmetries of $\mathrm{H}_{2}, \mathrm{D}_{2}$ or $\mathrm{T}_{2}$ hydrogen isotopes in their homo-molecular form, trapped as metastable states in the solid, complicate the polarization of the corresponding targets. However, HD, a heteromolecular form of hydrogen has no symmetry and can be polarized [16]. Static polarization, which requires high field $(\approx 15 \mathrm{~T})$ and very low temperature $(\approx 10 \mathrm{mK})$, has been achieved using distilled HD [17], opening the possibility to 
produce polarized DT molecules [18]. However, the static polarization of a DT sample is furthermore affected by the heat generated $\left(\approx 640 \mathrm{~mW} \mathrm{~g}^{-1}\right)$ via the tritium $\beta$-decay. Another possible approach is via the dynamic nuclear polarization (DNP) of solid DT. While more difficult [19] compared with the DNP of HD [20] this process can be carried out at a relatively high temperature $(\approx 1 / 2 \mathrm{~K})$ and at a more modest magnetic field intensity $(\approx 1 \mathrm{~T})$. At present, the polarization of hydrogen, deuterium or tritium atoms reaches a high level of about $90 \%$, while the polarization of frozen DT fuel is about $40 \%$. Therefore, while the polarization of DT fuel still requires significant developments in production technology it appears promising and quite possible in principle.

The use of polarized DT fuel was proposed for the first time in the context of a MCF reactor [2]. Shortly thereafter, the same concept was proposed for ICF systems by More [21]. It has also been estimated that during the short duration of an ICF implosion the depolarization of the fuel should be negligible $[21,22]$ and a possible experimental verification of the persistence of the polarization has been proposed [23].

An analytic estimation of the effect provided by an increase in the DT fusion cross-section on the gain of an ICF target was made by Rosen et al [24]. They modified the isobaric model of Meyer-ter-Vehn [25] (neglecting the hot-spot mass and assuming a power law for the fractional burn-up) and generalized it allowing the pressure to drop by a factor $\varepsilon$ from the hot-spot to the cold fuel, thus approaching the isochoric Kidder model [26]. Moreover, they included the factor $\delta$ in the model to take into account the increase in DT reactivity for polarized fuel and they found that the driver energy scales as $\delta^{-3}$ in order to achieve the maximum gain, $G_{\max }$. Thus, the reduction in the incident energy should be of about a factor of 3 assuming $\delta=1.5$. Numerical calculation of the implosion of a fully spin-polarized ICF target was performed by Pan and Hatchett [27] where they estimated a reduction of about a factor of 2 of the required driver energy for a given gain and capsule design. A study considering $\mathrm{D}-{ }^{3} \mathrm{He}$ spin polarized fuel was also performed by Honda et al [28].

In this paper, we consider the effect of spin polarization in more detail for the case of ICF, where a spherical capsule containing the DT thermonuclear fuel is irradiated by laser beams [29]. In direct and indirect drive fusion the capsule compression provides the ignition of the fusion reaction in a central hot-spot, allowing for the propagation of an efficient thermonuclear burn wave through a fraction $(\approx 1 / 3)$ of fuel, thus providing a high energy gain $(G \approx 100)$. In this paper, we assume that the DT fusion cross-section could be increased by up to $50 \%$ with a perfectly spin polarized fuel. In section 2 , we numerically analyse how the ignition conditions of a typical ICF target are modified, then in section 3, an extensive numerical study is performed in order to find, for a given direct drive target, the relation between absorbed energy and gain as a function of the parameter $\delta$.

\section{Modification of the ignition condition}

Hereafter we assume that the DT nuclear fusion cross-section $\sigma$ can be increased up to a maximum factor of $50 \%$ as a consequence of polarization of the nuclei. In order to characterize this for intermediate polarization cases the nuclear

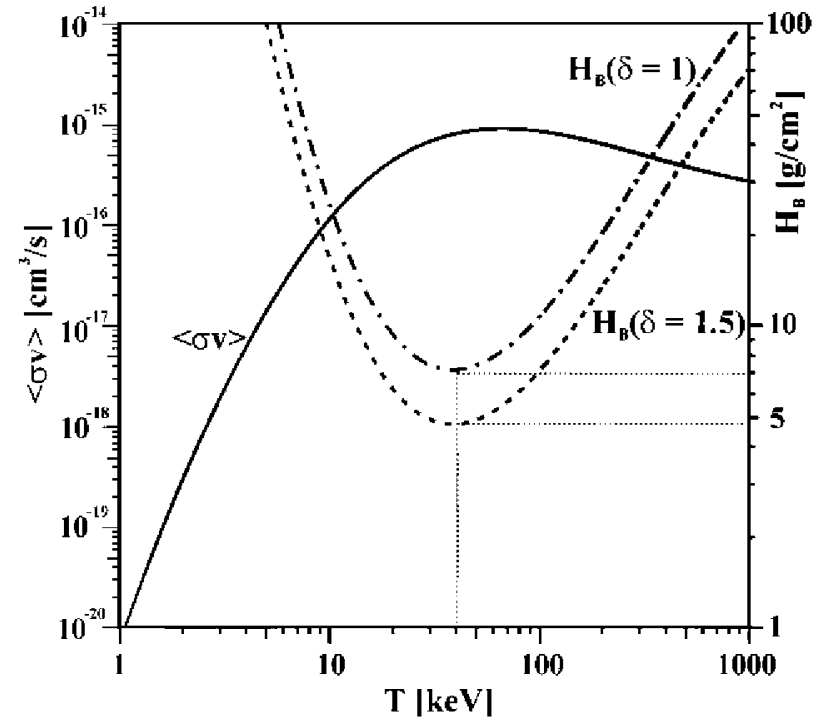

Figure 1. Average DT nuclear fusion reactivity $\langle\sigma v\rangle$ and burn parameter $H_{\mathrm{B}}$ as a function of the plasma temperature $T$.

fusion cross-section is multiplied by a factor $1 \leqslant \delta \leqslant 1.5$. First, the simplified case of a pre-compressed mass of DT is considered in order to analyse how the ignition conditions are modified by an increase in the fusion cross-section. For an equimolar DT fuel, the fusion reaction rate is proportional to the reactivity $\langle\sigma v\rangle$, which is the cross-section averaged over a Maxwellian velocity distribution. Bosch and Hale [30] provided a fit of the reactivity evaluated for a DT plasma characterized by a Maxwellian velocity distribution. This fit of the reactivity $\langle\sigma v\rangle$ is accurate in the range of temperature from $200 \mathrm{eV}$ to $100 \mathrm{keV}$, and is shown in figure 1 (solid line) as a function of the plasma temperature $T$. The burn efficiency (fractional burn-up) represents the fraction of the total DT mass that is burned via the thermonuclear fusion reactions. In the context of ICF, the burn efficiency is usually approximated [31] by $\phi=\rho R /\left(H_{\mathrm{B}}+\rho R\right)$, where $\rho R$ is the total fuel areal density and $H_{\mathrm{B}}$ is the burn parameter, which depends on the temperature $T$ and reactivity $\langle\sigma v\rangle$. The burn parameter can be approximated [8] by $H_{\mathrm{B}}\left(\mathrm{g} \mathrm{cm}^{-2}\right) \approx 9 T^{1 / 2} /\langle\sigma v\rangle$, where the fuel temperature and the reactivity are normalized to $100 \mathrm{keV}$ and $10^{-15} \mathrm{~cm}^{3} \mathrm{~s}^{-1}$, respectively. The burn parameter $H_{\mathrm{B}}$ is shown in figure 1 as a function of the fuel temperature for the usual DT cross-section $\delta=1$ (the dashed-dotted red curve) as well as for the increased cross-section with the parameter $\delta=1.5$ (the dashed green curve). As can be seen, the burn parameter $H_{\mathrm{B}}$ has a minimum of about $H_{\mathrm{B}} \cong 7$ at the optimum temperature of about $T \cong 40 \mathrm{keV}$ for the standard DT fuel $(\delta=1)$, while the minimum burn parameter becomes $H_{\mathrm{B}} \approx 5$ if the cross-section increases by $50 \%(\delta=1.5)$. Given that the burn parameter scales as $1 /\langle\sigma v\rangle$ it turns out that for a factor $\delta$ larger than one a reduced areal density is sufficient in order to produce the same number of fusion reactions. In fact, a total fuel confinement $\rho R=3 \mathrm{~g} \mathrm{~cm}^{-2}$ is needed to burn approximately $\phi=30 \%$ of the DT fuel mass with $\delta=1$ $\left(H_{\mathrm{B}}=7\right)$, while for the same parameters the burn efficiency grows to $\phi \approx 37 \%$ assuming a factor $\delta=1.5\left(H_{\mathrm{B}}=5\right)$.

The energy gain $G=E_{\mathrm{TN}} / E$ of an ICF capsule is proportional to the burn efficiency. In fact, $G=q_{\mathrm{DT}} M_{\mathrm{DT}} \phi / E$, 
where $q_{\mathrm{DT}}=3.34 \times 10^{11} \mathrm{~J} \mathrm{~g}^{-1}, M_{\mathrm{DT}}$ is the total DT mass, $E$ is the energy delivered by the driver and $E_{\mathrm{TN}}$ is the thermonuclear energy produced by the fusion reactions. Therefore, if the cross-section growth by a factor $\delta$ implies a decrease in the burn parameter $H_{\mathrm{B}}(\propto 1 / \delta)$, this in turn allows for higher fractional burn-up $\phi$ and consequently higher gains. Moreover, for a fixed fuel mass, we will show that there will be a reduction in the total invested energy $E$ because of the reduced temperature and density required to achieve ignition, which implies an additional increase in the energy gain.

The classical central hot-spot ignition ICF scheme assumes that a spherical target is compressed generating a small central volume characterized by a relatively small density $\rho_{\mathrm{h}}<100 \mathrm{~g} \mathrm{~cm}^{-3}$ and a high temperature $T_{\mathrm{h}}$ of several keV where the ignition starts. This hot mass, usually called a hotspot, is confined by a relatively cold shell at high density $\rho_{\mathrm{c}}$ (hundreds of $\mathrm{g} \mathrm{cm}^{-3}$ ) and low temperature $T_{\mathrm{c}} \ll T_{\mathrm{h}}$. In this configuration the fuel is nearly isobaric. For some values of the confinement parameter $\rho_{\mathrm{h}} R_{\mathrm{h}}$ and temperature $T_{\mathrm{h}}$ this configuration allows for the self-ignition of the fusion reactions followed by successful thermonuclear burn wave propagation. A set of numerical simulations have been performed with the mono-dimensional (1D) hydro-radiative code MULTI-fs $[32,33]$ in order to find the region in the $\rho_{\mathrm{h}} R_{\mathrm{h}}-T_{\mathrm{h}}$ parameter space where the fuel configuration provides a successful ignition. In these calculations a successful ignition is defined by both an increased mass averaged temperature within the original hot-spot radius, $T_{\mathrm{HS}}$, and an increased confinement parameter in the heated plasma, $(\rho R)_{\mathrm{HS}}$. Here, the heated plasma is defined by the region with temperatures higher than $T=\operatorname{Min}\left(4 \mathrm{keV}, T_{\mathrm{HS}} / 2\right)$. Therefore, a successful ignition occurs if at some later time both the temperature and confinement parameter exceed their original values, i.e. $T_{\mathrm{HS}}>T_{\mathrm{h}}$ and $(\rho R)_{\mathrm{HS}}>\rho_{\mathrm{h}} R_{\mathrm{h}}$. In these calculations, the density $\rho_{\mathrm{h}}$ and the temperature $T_{\mathrm{h}}$ of the hot-spot are the input data, while the hot-spot radius is set to $R_{\mathrm{h}}=100 \mu \mathrm{m}$, the density of the cold shell is $\rho_{\mathrm{c}}=16 \rho_{\mathrm{h}}$ and the shell temperature $T_{\mathrm{c}}=T_{\mathrm{h}} / 16$. According to the conditions of spark formation [34] the available parametric space is bounded at high temperatures $T_{\mathrm{h}}$ and at large $\rho_{\mathrm{h}} R_{\mathrm{h}}$ (see the shaded areas in figure 2). In fact, hot-spots with a very large confinement parameter $\rho_{\mathrm{h}} R_{\mathrm{h}}$ cannot be assembled due to the energy losses via bremsstrahlung radiation giving the condition $\rho_{\mathrm{h}} R_{\mathrm{h}}\left(\mathrm{g} \mathrm{cm}^{-2}\right)<0.12 T_{\mathrm{h}}(\mathrm{keV})$, while hot-spots that are very small are inhibited by the electron thermal conductivity leading to the requirement $\rho_{\mathrm{h}} R_{\mathrm{h}}>8 \times 10^{-3} T_{\mathrm{h}}^{2} / \ln \Lambda$, where $\ln \Lambda$ is the Coulomb logarithm.

The parameters that allow for self-ignition in the case of the usual DT fuel $(\delta=1)$ are shown as red spots in figure 2 , while the empty blue circles indicate the non-igniting areas considering an increased fusion cross-section with a factor $\delta=1.5$. Thus, the increased cross-section provides a larger ignition parameter space and the void space in figure 2 indicates the new region now available for ignition when $\delta=1.5$. It is found that the two parameters $\rho_{\mathrm{h}} R_{\mathrm{h}}$ and $T_{\mathrm{h}}$, which characterize the hot-spot located at the border of the igniting area, can both be reduced by more than $15 \%$ as a consequence of the increased DT nuclear fusion cross-section $(\delta=1.5)$. The void circle (A) indicates the position of a typical self-igniting hotspot for an isobaric DT fuel configuration and $\delta=1$. Thus,

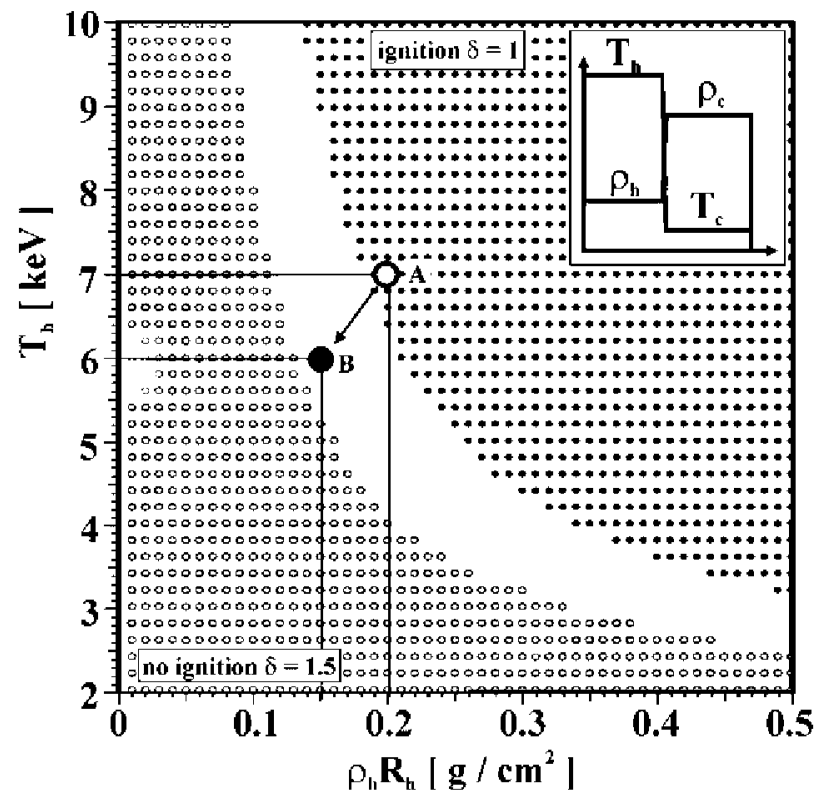

Figure 2. Igniting area for $\delta=1$ (red spots) and non-igniting area for $\delta=1.5$ (empty blue circles) in the parameter space $\rho_{\mathrm{h}} R_{\mathrm{h}}-T_{\mathrm{h}}$.

the confinement parameter $\rho_{\mathrm{h}} R_{\mathrm{h}}=0.2 \mathrm{~g} \mathrm{~cm}^{-2}$ and the hotspot temperature $T_{\mathrm{h}}=7 \mathrm{keV}$ (A) could be reduced to about $0.15 \mathrm{~g} \mathrm{~cm}^{-2}$ and $6 \mathrm{keV}(\mathrm{B})$, respectively, assuming $\delta=1.5$.

These data are used as input in an isobaric model [25] of a pre-compressed, self-igniting hot-spot to estimate the energy gain $G\left(E, T_{\mathrm{h}}, \rho_{\mathrm{h}}, R_{\mathrm{h}}, \eta \alpha\right)$, where $E$ is the total invested energy, $\eta$ is the hydrodynamic efficiency and $\alpha$ is the isentropic parameter. In our example, the hot-spot radius is $R_{\mathrm{h}}=100 \mu \mathrm{m}$ and the hydrodynamic efficiency is set to be $\eta=15 \%$, while the isentropic parameter is taken as $\alpha=2$. The energy gains $G_{\mathrm{A}}(E)$ and $G_{\mathrm{B}}(E)$ are calculated for the parameters of case A $\left(\delta=1, H_{\mathrm{B}}=7 \mathrm{~g} \mathrm{~cm}^{-2}\right)$ and case $\mathrm{B}\left(\delta=1.5, H_{\mathrm{B}}=5 \mathrm{~g} \mathrm{~cm}^{-2}\right)$, and are shown in figure 3 as a function of the driver energy $E$. It is found that for an invested energy $E=1 \mathrm{MJ}$ the estimated gains are $G_{\mathrm{A}}(1 \mathrm{MJ}) \approx 130$ for $\delta=1$ while $G_{\mathrm{B}}(1 \mathrm{MJ}) \approx 300$ when the cross-section factor is increased by a factor $\delta=1.5$. Moreover, for a fixed gain $G$, the ratio $E_{\mathrm{B}} / E_{\mathrm{A}}$ is always smaller than 0.7 , decreasing as the gain increases (see the dashed line in figure 3 ), and indicates that the required invested energy $E_{\mathrm{B}}$ decreases as the factor $\delta$ increases. As an example, for a gain $G \approx 100$ the driver energy $E_{\mathrm{A}} \approx 880 \mathrm{~kJ}$ with $\delta=1$, while a smaller energy $E_{\mathrm{B}} \approx 510 k J\left(E_{\mathrm{B}} / E_{\mathrm{A}} \approx 0.58\right)$ is needed assuming $\delta=1.5$.

The minimum energy needed to heat the hot-spot mass

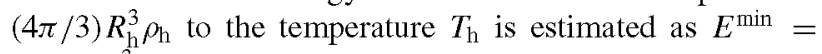
$4 \pi k T_{\mathrm{h}} R_{\mathrm{h}}^{3} \rho_{\mathrm{h}} /\left(\eta 2.5 m_{\mathrm{p}}\right)$, where $m_{\mathrm{p}}$ is the proton mass. This minimum energy scales as $E^{\min } \propto T_{\mathrm{h}} R_{\mathrm{h}} \rho_{\mathrm{h}}$ and therefore a reduction of $15 \%$ in both parameters $T_{\mathrm{h}}$ and $R_{\mathrm{h}} \rho_{\mathrm{h}}$ implies a reduction of about $30 \%$ in $E^{\mathrm{min}}$. In our cases, these minimum energies evaluated by the isobaric model correspond to $E_{\mathrm{A}}^{\min } \approx$ $450 \mathrm{~kJ}$ and $E_{B}^{\min } \approx 290 \mathrm{~kJ}$.

\section{1D numerical simulations}

A set of numerical simulations are performed with the MULTI-fs 1D radiation hydrodynamics code $[32,33]$, which 


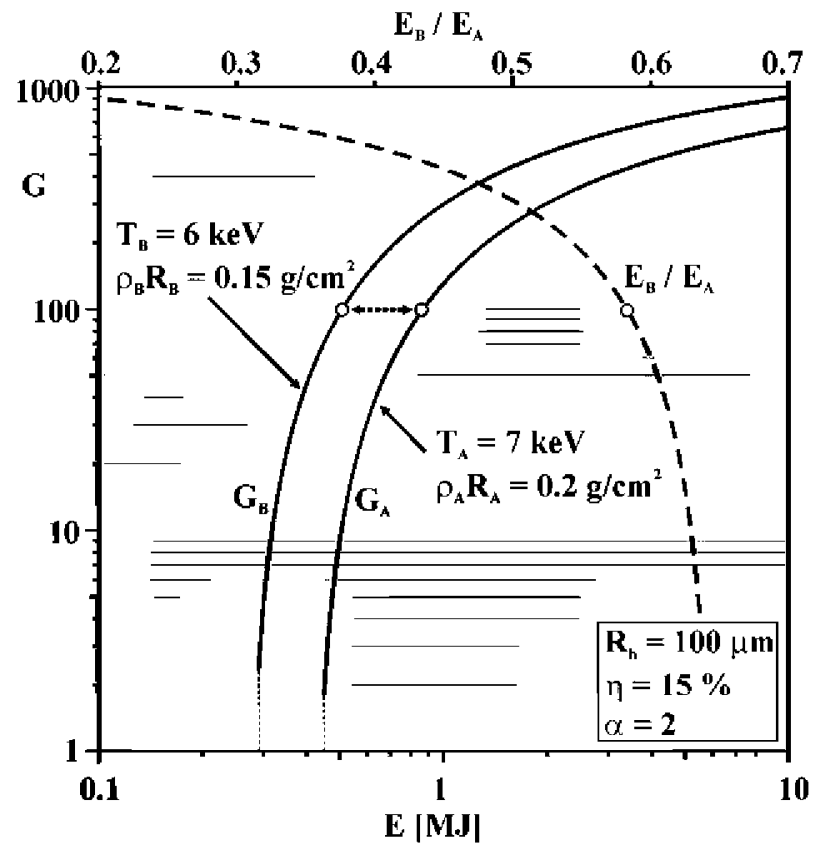

Figure 3. Isobaric model: energy gain $G$ as a function of the driver energy $E$ (solid lines) and energy ratio $E_{\mathrm{B}} / E_{\mathrm{A}}$ as a function of gain $G$ (dashed line).

uses tabulated equations of state (SESAME), thermal heat conduction with flux harmonically limited to $8 \%$ and a 3D raytracing treatment of the laser-capsule interaction. A capsule [35] recently designed in the context of the French Laser MegaJoule (LMJ) facility $[36,37]$ is considered. For this specific design it is assumed that the capsule will be directly irradiated [38] by the laser beams. The capsule is composed of an external shell of plastic $\mathrm{CH}\left(\rho_{\mathrm{CH}}=1.07 \mathrm{~g} \mathrm{~cm}^{-3}\right)$ that encloses $300 \mu \mathrm{g}$ of a cryogenic DT nuclear fuel $\left(\rho_{\mathrm{DT}}=\right.$ $0.25 \mathrm{~g} \mathrm{~cm}^{-3}$ ). The initial aspect ratio of this capsule is equal to 3 , the external radius of the capsule is $r_{0}=815 \mu \mathrm{m}$ while the shell of $\mathrm{CH}$ is $24 \mu \mathrm{m}$ thick and is used to absorb the laser energy. The capsule is directly irradiated by laser beams characterized by a Gaussian intensity profile with a full-width at half-maximum (FWHM) of $1356 \mu \mathrm{m}$, thus the laser intensity is reduced to $1 / e$ at the initial capsule radius $r_{0}$. The evolution of the Lagrangian capsule radii as a function of time is shown in figure 4 . In this case, the temporal profile of the laser incident power maximizes the final energy gain, which is $G_{\max }=64$, while the factor $\delta$ is set to be 1.5 .

In order to maximize the final gain a large number of simulations are performed randomly choosing the incident laser intensity profile. For this purpose, the laser pulse is designed using a seven-point piecewise linear function for the power as a function of time given by powers $P_{i}$ defined at the corresponding times $t_{i}(i=1-7)$. In the two frames of figure 5 the randomly selected laser intensity profiles (blue lines) that provide a gain of larger than $0.9 G_{\max }$ using $\delta=1$ (left) and $\delta=1.5$ (right) are shown. A set of a thousand simulations are performed by randomly selecting the pairs $\left[t_{i}, P_{i}\right]$. The continuous black line represents the incident laser profiles that realize the largest gains. It is found that with $\delta=1$ this capsule generates a maximum gain of about $G_{\max }=44$ with an incident laser energy of about $500 \mathrm{~kJ}$, while the gain grows

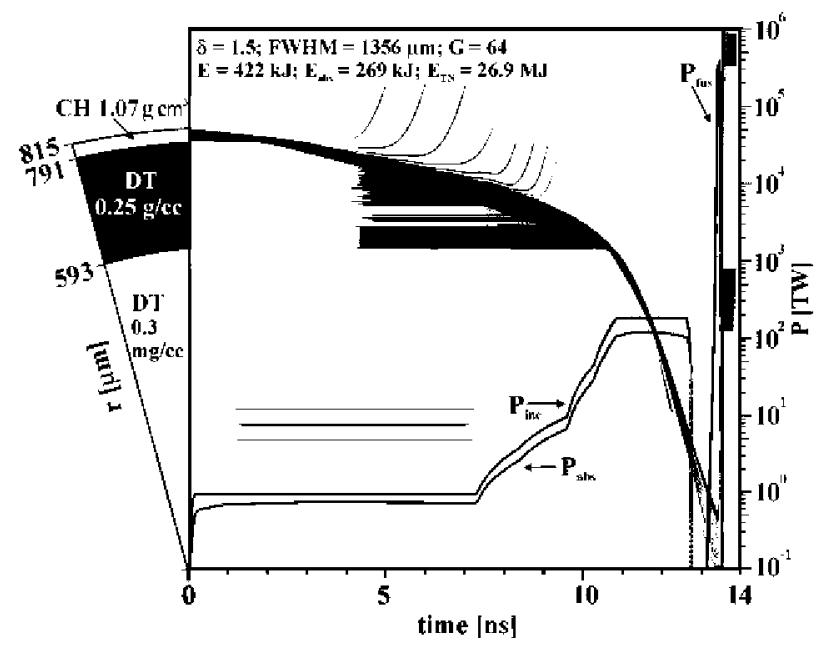

Figure 4. Temporal evolution of the Lagrangian radii evaluated with $\delta=1.5$. The laser incident power (blue line), the absorbed power (green line) and the fusion produced power (black line) are also shown as a function of time.

to $G_{\max }=64$ and the incident laser energy decreases to $420 \mathrm{~kJ}$ when a factor $\delta=1.5$ is assumed.

In figure 6 (left) the energy gain $G$ as a function of the maximum of the laser power $\left(P_{\max }\right)$ is shown, while in figure 6 (right) the gain versus the laser absorbed energy $E_{\text {abs }}$ is shown. The red and blue points correspond to the simulations with $\delta=1$ and $\delta=1.5$, respectively. As can be seen in figure 6 , a relatively sharp transition separates the non-igniting and igniting cases. The transition between the two regimes, shown by vertical dashed lines in figure 6 , is characterized by a minimum power $P^{*}$ and a minimum absorbed energy $E_{\mathrm{abs}}^{*}$ that has been defined where the gain becomes larger than $0.9 G_{\max }$. For this capsule, the calculations performed with the standard DT reactivity $(\delta=1)$ show that this transition occurs at about $P^{*} \approx 215 \mathrm{TW}, E_{\mathrm{abs}}^{*} \approx 290 \mathrm{~kJ}$, while those with the increased reactivity $(\delta=1.5)$ exhibit a lower threshold around $P^{*} \approx 170 \mathrm{TW}$ and $E_{\mathrm{abs}}^{*} \approx 250 \mathrm{~kJ}$.

A set of calculations are also carried out assuming $\delta=1.1$, 1.2, 1.3 and 1.4. From all the datasets, the maximum gain $G_{\max }$, the threshold power $P^{*}$ and the threshold absorbed energy $E_{\text {abs }}^{*}$ are extracted and they are shown in figure 7 as a function of the parameter $\delta$. As shown in figure 7, the growth of the fusion cross-section implies an increase in the thermonuclear energy gain while the required invested energy is reduced. Moreover, it is confirmed that the maximum gain scales as $\delta^{0.9}$ [24], while both the minimum required power $P^{*}$ and the absorbed energy $E_{\text {abs }}^{*}$ scale approximately as $\delta^{-0.6}$ and $\delta^{-0.4}$, respectively.

\section{Conclusions}

The positive effect provided by the increase in the fusion crosssection by a factor $\delta$ as a consequence of the spin polarization of the DT nuclei is investigated. Considering an isobaric ICF fuel configuration it is shown that the self-igniting parametric space becomes larger when a factor $\delta=1.5$ is assumed. Thus, the required ignition conditions are relaxed and the threshold values of the areal density and temperature can both be reduced by more than $15 \%$. As a consequence, the isobaric model 

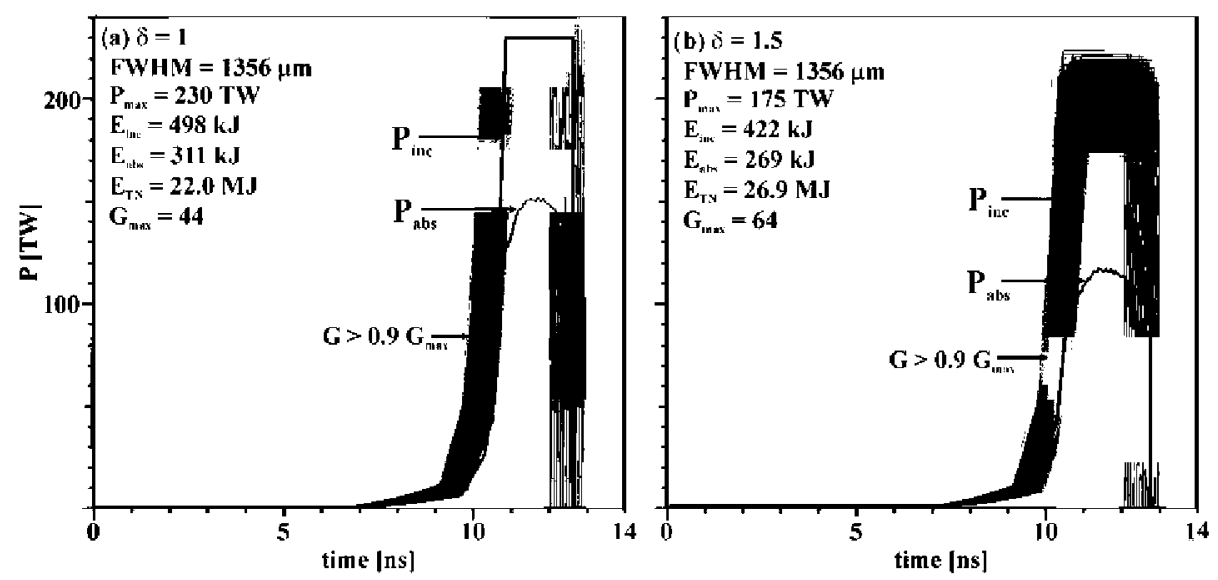

Figure 5. Temporal profiles of the laser power that produce a gain $G \geqslant 0.9 G_{\max }$ (blue lines). The continuous black lines show the incident power profile $\left(P_{\text {inc }}\right)$ that realizes the maximum gain as well as the associated absorbed power ( $P_{\text {abs }}$, green lines).
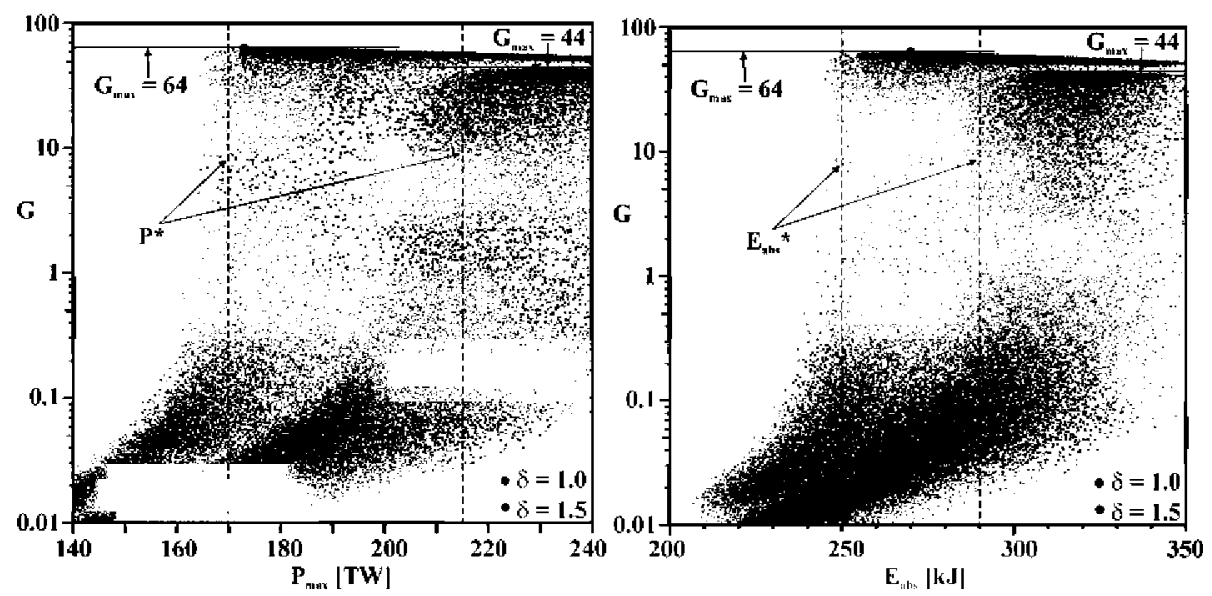

Figure 6. Gain as a function of the maximum of the incident laser power $P_{\max }(l \mathrm{eft})$ and of the laser absorbed energy $E_{\mathrm{abs}}(\mathrm{right})$.

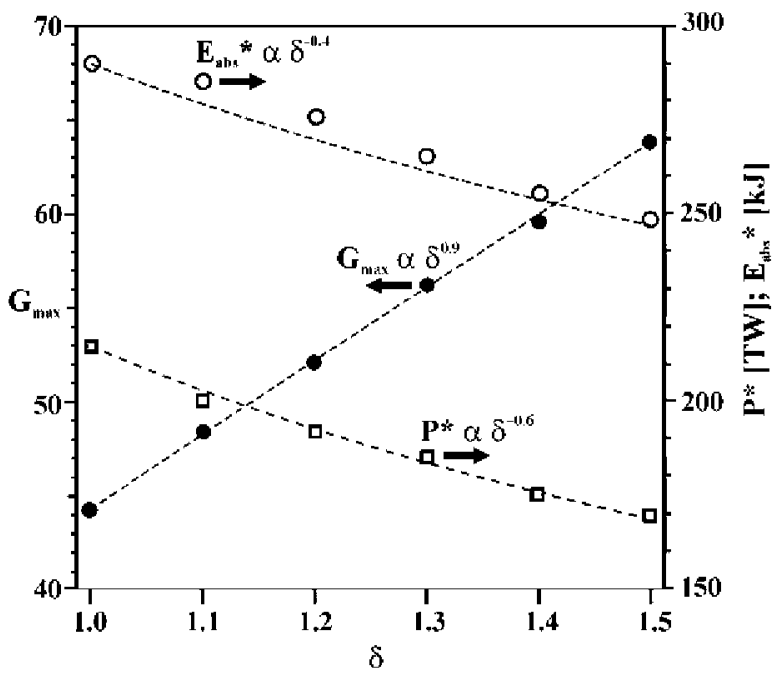

Figure 7. Maximum gain $G_{\max }$ (full circles), minimum incident power $P^{*}$ (squares) and absorbed energy $E_{\text {abs }}^{*}$ (void circles) as a function of the factor $\delta$.

shows that the required invested energy is reduced by more than $30 \%$ and decreases as the gain increases. Moreover, a detailed numerical study is performed for a direct drive capsule while varying the factor $\delta$. It is confirmed that the maximum gain scales as $G_{\max } \propto \delta^{0.9}$, as found by Rosen et al [24], while the ignition threshold of the incident laser power decreases as $\delta^{-0.6}$ and the absorbed energy decreases by the factor $\delta^{-0.4}$. Thus, for fully polarized DT fuel ( $\delta=1.5$ ), the maximum gain increases by about $45 \%$ while the required laser power and the absorbed energy decrease by about $20 \%$ and $15 \%$, respectively.

The advantages gained by using polarized fuel could be quite significant and it is expected that this technique could be particularly important for systems operating close to the ignition threshold. Not only are the required laser energies reduced, but the total yield in fusion energy can also be reduced, which in turn would reduce the loading and stress in real reactor designs. At the same time, it is found that considerable advantages are obtained in terms of lower energy requirements, higher gain, lower areal density and temperature required for ignition. Moreover, the implosion velocity and in-flight aspect ratio of the enhanced cross-section fuel are reduced [39] compared with the unpolarized DT leading to more robust implosions, thus reducing the sensitivity to hydrodynamic instabilities such as the Rayleigh-Taylor instability. Nevertheless, further investigations are required to establish whether such spin-polarized fuel can be fabricated and maintained during the interaction process such that these benefits can be realized. 


\section{Acknowledgments}

M.T. was supported by the research Contract 'Ramon y Cajal' 2007-00447 of the Spanish Ministry of Education and Research (MEC). R.R. was partially supported by the EURATOM/CIEMAT association in the framework of the 'IFE Keep-in-Touch Activities'. Support from the contract ENE2009-11668 of the MEC is also acknowledged.

\section{References}

[1] Conner J.P., Bonner T.W. and Smith J.R. 1952 Phys. Rev. 88468

[2] Kulsrud R.M., Furth H.P., Valeo E.J. and Goldhaber M. 1982 Phys. Rev. Lett. 491248

[3] Takatsu H. 2011 Nucl. Fusion 51094002

[4] Nuckolls J.H., Wood L., Thiessen A. and Zimmermann G.B. 1972 Nature 239129

[5] Lindl J. 1995 Phys. Plasmas 23933

[6] Lindl J.D. 1998 Inertial Confinement Fusion: The Quest for Ignition and High Gain Using Indirect Drive (New York: Springer)

[7] Lindl J.D., Amendt P., Berger R.L., Glendinning S.G., Glenzer S.H., Haan S.W., Kauffman R.L., Landen O.L. and Suter L.J. 2004 Phys. Plasmas 11339

[8] Atzeni S. and Meyer-ter-Vehn J. 2004 The Physics of Inertial Fusion (Oxford: Oxford University Press)

[9] Kulsrud R.M., Valeo E.J. and Cowley S.C. 1986 Nucl. Fusion 261443

[10] Souers P.C., Fearon E.M., Mapoles E.R., Gaines J.R., Sater J.D. and Fedders P.A. 1986 J. Vac. Sci. Technol. A 41118

[11] Souers P.C. and Fedders P.A. 1990 Phys. Rev. B 418643

[12] Paetz gen. Schieck H. 2010 Eur. Phys. J. A 44321

[13] Wise T. 2005 Proc. 11th Int. Workshop on Polarized Sources and Targets (Tokyo, Japan) ed T. Uesaka et al (Singapore: World Scientific) pp 11-7

[14] Nass A. et al 2003 Nucl. Instrum. Methods Phys. Res. A $\mathbf{5 0 5} 633$

[15] Toporkov D. et al 2011 Proc. 14th Int. Workshop on Polarized Sources, Targets and Polarimetry (St Petersburg, Russia) ed K. Grigoryev et al $\mathrm{p} 73$

[16] Honig A. 1967 Phys. Rev. Lett. 191009
[17] Bouchigny S., Didelez J.P., Dubois F. and Rouillé G. 2009 Nucl. Instrum. Methods Phys. Res. A 607271

[18] Bouchigny S. and Didelez J.P. 2007 Nucl. Instrum. Methods Phys. Res. A $\mathbf{5 7 7} 320$

[19] Souers P.C., Tsugawa R.T., Fearon E.M., Collins G.W., Maienschein J.L., Failor R.A., Mapoles E.R. and Fedders P.A. 1993 J. Vac. Technol. A 112827

[20] Solem J.C. 1974 Nucl. Instrum. Methods Phys. Res. A 117477

[21] More R.M. 1983 Phys. Rev. Lett. 51396

[22] Goel B. and Heeringa W. 1988 Nucl. Fusion 28355

[23] Didelez J.P. and Deutsch C. 2011 Laser Part. Beams 29169

[24] Rosen M.D., Lindl J.D. and Thiessen A.R. 1983 Laser Program Annual Report UCRL-50021-83, Lawrence Livermore National Laboratory, Lawrence pp 3-5-3-9

[25] Meyer-ter-Vehn J. 1982 Nucl. Fusion 22562

[26] Kidder R.E. 1976 Nucl. Fusion 16405

[27] Pan Y. and Hatchett S.P. 1987 Nucl. Fusion 27815

[28] Honda T., Nakao Y., Honda Y., Kudo K. and Nakashima H. 1991 Nucl. Fusion 31851

[29] Nuckolls J.H., Wood L., Thiessen A. and Zimmerman G.B. 1972 Nature 239129

[30] Bosch H.S. and Hale G.M. 1992 Nucl. Fusion 32611

[31] Fraley G.S., Linnebur E.J., Mason R.J. and Morse R.L. 1974 Phys. Fluids 17474

[32] Ramis R., Schmaltz R. and Meyer-ter-Vehn J. 1988 Comput. Phys. Commun. $\mathbf{4 9} 475$

[33] Ramis R., Eidmann K., Meyer-ter-Vehn J. and Hüller S. 2012 Comput. Phys. Commun. 183637

[34] Basko M.M. 1990 Nucl. Fusion 302443

[35] Brandon V., Canaud B., Laffite S., Temporal M. and Ramis R. 2011 Systematic analysis of direct-drive baseline designs for shock ignition with the laser MegaJoule Proc. 7th Conf. on Inertial Fusion Sciences and Applications

(Bordeaux-Lac, France, 12-16 September 2011) (European Physical Journal: Web of Conferences)

[36] Cavailler C. 2005 Plasma Phys. Control. Fusion 47 B389

[37] Giorla J. et al 2006 Plasma Phys. Control. Fusion 48 B75

[38] Canaud B. et al 2007 Plasma Phys. Control. Fusion 49 B601

[39] Temporal M., Brandon V., Canaud B., Didelez J.P., Fedosejevs R. and Ramis R. 2012 Investigation of the ignition conditions of polarized DT fuel for inertial confinement fusion 39th European Physical Society Conf. on Plasma Physics (Stockholm, Sweden, 2-6 July 2012) and http://ocs.ciemat.es/epsicpp2012pap/pdf/P2.111.pdf 